\title{
Rousseau and His Team's Study on Gefura Measures
}

\author{
Li Weigang \\ TransLab, Department of Computer Science, University of Brasilia, Brasilia-DF, Brazil \\ Email: weigang@unb.br
}

Received 9 April 2015; accepted 22 April 2015; published 29 April 2015

Copyright (C) 2015 by author and Scientific Research Publishing Inc.

This work is licensed under the Creative Commons Attribution International License (CC BY). http://creativecommons.org/licenses/by/4.0/

(c) (i) Open Access

\begin{abstract}
Guns and Rousseau's recently published paper in FITEE gives a clear introduction about some important research results related to complex networks, which deepens people's understanding of network characteristics, and puts forward new measures and models. The proposed gefura measures show the significance of appropriately using a "basic" normalization to describe the betweenness centrality of nodes, and then the "structural" normalization to pay more attention to the level of groups. The term "gefura measures" is from old Greek $\gamma \varepsilon \varphi v \rho \alpha$, meaning bridge measure, a more descriptive term with universal appeal. More specifically, they applied the Brandes algorithm to calculate the gefura measures, which makes their article easier to apply to practical cases. Even in academia, any new ideas, concepts, indicators and models should stand the test of time. The spirit of innovation of Rousseau and his team is highly recommendable. We sincerely hope that the study on gefura measures can raise more concerns, and can be recognized and used by social scientists, informetricians, and colleagues studying complex networks from all over the world as an important outcome.
\end{abstract}

\section{Keywords}

Complex Networks, Follow Model, Gefura Measures, Q-Measure, Rousseau

In the latest issue Vol. 16, No. 4 (April 2015), the journal Frontiers of Information Technology \& Electronic Engineering published a paper entitled "Unnormalized and Normalized Forms of Gefura Measures in Directed and Undirected Networks” [1] from the scientometrician Ronald Rousseau and his team. Because of similar research direction, we have the chance to study more about the process how the authors proposed and designed their research on gefura measures. Here, I would like to recommend this research work to the readers from the community of complex networking and related fields.

In this paper [1], Guns and Rousseau give us a very clear introduction about some important research results 
related to complex networks, which deepens people's understanding of network characteristics, and put forward new measures and models. The paper reviews the investigations about brokerage [2], betweenness centrality [3], [4], Q-measures [5], and Rousseau and his team's study on gefura measures. Furthermore, researchers designed some important indicators, and proposed relevant models and algorithms to characterize or calculate the indicators. For example, Brandes [6] (2001) introduced a fast algorithm to determine betweenness centrality. The term "gefura measures" is from old Greek $\gamma \varepsilon \varphi v \rho \alpha$, meaning bridge measure.

As complex networks are often multiple heterogeneous with distinct levels, nodes normally express the basic elements, and the group consisting of a set of nodes expresses a high-level generic element. In some networks, nodes belong to predefined groups, and we can know some internal relations. However, common network centrality measures do not take this structure into account and cannot show the changes of this structure. Gefura measures proposed by Rousseau and his team show the significance of appropriately using a "basic" normalization to describe the betweenness centrality of nodes, and then the "structural" normalization to pay more attention to the level of groups. Though researchers started by a systematic study in directed networks, the undirected case follows straightforwardly by treating undirected networks as symmetric directed networks. Moreover, normalization makes it possible to compare gefura measures across networks of different sizes. More specifically, Guns and Rousseau applied the Brandes algorithm [6] to calculate their gefura measures, which makes this article easier to apply to practical cases.

In the field of scientometrics, literature network analysis may have articles or authors as nodes, and journals or conferences as groups. Through gefura measures, authors or articles' bridge role between groups will be clearer. Particularly, using basic gefura measures and structural gefura measures can distinguish the domestic citations and international citations, reflecting the real value of the research articles, which shows a profound significance in practical applicability. Figure 1 shows the screenshot of this article. For full text, please visit the journal website of FITEE [1].

In academia, any new ideas, concepts, indicators and models should stand the test of time. Professor Rousseau and his team formally put forward "gefura measures" by publishing their article in FITEE. This spirit of innovation is highly recommendable. In fact, the evolution from Q-measures to gefura measures has taken a long time. In the past ten years, Professor Rousseau and his team have always adhered to the direction of this research and now we see the integration of their study on gefura measure. Q-measures as the indicators of brokerage have a restriction that the definition allowed for only two node groups. This restriction was removed by Guns and Rousseau [7] (2009), who generalized the Q-measure definition to any finite number of groups and introduced the distinction between "global" Q-measures (brokerage between all different groups) and "local” Q-measures (brokerage between one's own group and the other groups). The SCIENCENET blogger Liu Yuxian took part in

\title{
Unnormalized and normalized forms of gefura measures in directed and undirected networks*
}

\author{
Raf GUNS ${ }^{1}$, Ronald ROUSSEAU $\$ 1,2$ \\ ('Institute for Education and Information Sciences, University of Antwerp, Venusstraat 35, Antwerp B-2000, Belgium) \\ ( 2 Department of Mathematics, KU Leuven, Celestijnenlaan 200B, Leuven B-3001, Belgium) \\ E-mail: raf.guns@uantwerpen.be; ronald.rousseau@kuleuven.be; ronald.rousseau@uantwerpen.be \\ Received Dec. 9, 2014; Revision accepted Mar. 12, 2015; Crosschecked Mar. 13, 2015
}

Figure 1. Screenshot of Guns and Rousseau’s article published in FITEE, April 2015. 
some research work [8] [9] from 2010 to 2015, which related to mathematical properties, normalization, weight settings and application in co-authorship networks. Considering that using the symbol Q and the term Q-measure to study brokerage is not optimal at all, Professor Rousseau and his team specifically proposed "gefura measures”, a more descriptive term with universal appeal.

For local gefura measures, Guns and Rousseau proposed three cases for describing the nodes' brokerage role: outward bridge, inward bridge, both outward and inward bridges. This enables the formula for gefura measures to be expressed concisely and accurately. For the past years, our team focused on the Follow Model by using a function to describe the relationship in different users (followee, follower and both friends) [10] [11]. The original intention of our study is to present the basic relationship between users in online social networks and to explore an efficient algorithm for querying information fast. After studying the gefura measures, I think it will further enhance the depth and breadth of our research on microblog, if we can combine the advantages of the Follow Model and gefura measures.

Actually, the study related to complex networks, such as describing the characteristics of individuals, showing the formation and evolution of groups, analysing the key brokerage and variable feature between groups, is a very interesting and important subject. To make it popular and easy to understand, we can take the introduction of talented scientists as an example. If a talented scholar is introduced properly, he/she will bring the previous team(s)' scientific research resources to the new team, become the connection of multiple groups, and promote academic exchanges and research development of different teams. Then the gefura measures can be used to describe the impact and academic value of this colleague.

The SCIENCENET blogger Wu Yishan once introduced the development timelines of scientometrics compiled by Professor Rousseau, which showed that major achievements have not yet appeared in this field after 2005 [12]. We sincerely hope the study on gefura measures can raise more concerns, and can be recognized and used by social scientists, informetricians, and colleagues studying complex networks from all over the world as an important outcome. On the other hand, this study on gefura measures can only be applied to the static network at present, as for the case under dynamic conditions, the connection of nodes and groups should be discussed further. Researchers can find room to make in-depth study related to this topic. For instance, another SCIENCENET blogger Zhou Tao [13] recommended an article from Cheng Can et al. [14], which is related to the transmission strategy of network routing and pays more attention to the dynamic connection. All roads lead to Rome. It is worth learning from relevant research results.

It should be noted that the publication of this article also reflects the efforts from FITEE Editorial Office to promote the internationalization of Chinese domestic journals. Introducing domestic research achievements to the world and publishing the research articles from international famous scholars have become the daily work of this international journal. In early 2015, FITEE, formerly known as Journal of Zhejiang University-SCIENCE C (Computers \& Electronics), became one of the transactions of the Chinese Academy of Engineering (CAE), and then it was indexed by Web of Science from the January issue. Returning to the article from Professor Rousseau and his team, researchers can also learn the skills of writing a scientific research paper and guide students to apply the adapted Brandes algorithm to do some case studies.

\section{Acknowledgements}

This communication is re-organized from my Chinese blog from SCIENCENET [15]. Thanks the translation work by Ms. Yaqin Yan and the comments from Professor Ronald Rousseau and Mr. Zhai Ziyang.

\section{References}

[1] Guns, R. and Rousseau, R. (2015) Unnormalized and Normalized Forms of Gefura Measures in Directed and Undirected Networks. Frontiers of Information Technology \& Electronic Engineering, 16, 311-320. http://dx.doi.org/10.1631/FITEE.1400425

[2] Gould, R.V. and Fernandez, R.M. (1989) Structures of Mediation: A Formal Approach to Brokerage in Transaction Networks. Sociological Methodology, 19, 89-126. http://dx.doi.org/10.2307/270949

[3] Freeman, L.C., Borgatti, S.P. and White, D.R. (1991) Centrality in Valued Graphs: A Measure of Betweenness Based on Network Flow. Social Network, 13, 141-154. http://dx.doi.org/10.1016/0378-8733(91)90017-N

[4] Wasserman, S. and Faust, K. (1994) Social Network Analysis: Methods and Applications. Cambridge University Press, Cambridge, UK. http://dx.doi.org/10.1017/CBO9780511815478 
[5] Flom, P.L., Friedman, S.R. and Strauss, S. (2004) A New Measure of Linkage between Two Sub-Networks. Connections, 26, 62-70.

[6] Brandes, U. (2001) A Faster Algorithm for Betweenness Centrality. Journal of Mathematical Sociology, 25, $163-177$. http://dx.doi.org/10.1080/0022250X.2001.9990249

[7] Guns, R. and Rousseau, R. (2009) Gauging the Bridging Function of Nodes in a Network: Q-Measures for Networks with a Finite Number of Subgroups. Proceedings of 12th International Society for Scientometrics and Informetrics (ISSI), Vienna, July 2012, 131-142.

[8] Guns, R. and Liu, Y.X. (2010) Scientometric Research in China in Context of International Collaboration. Proceedings of 6th International Conference on Scientometrics and University Evaluation, Wuhan, November 2010, 112-115.

[9] Liu, Y.X., Guns, R. and Rousseau, R. (2013) A Binary Tree as a Basic Model for Studying Hierarchies Using Q-Measures. SRELS Journal of Information Management, 50, 521-528.

[10] Sandes, E.F.O, Weigang, L. and Melo, A.C.M. (2012) Logical Model of Relationship for Online Social Networks and Performance Optimizing of Queries. Proceedings of the 13th International Conference on Web Information Systems Engineering, Paphos, Cyprus, 28-30 November 2012, 726-736.

[11] Weigang, L., Sandes, E.F.O., Zheng, J., Melo, A.C.M. and Uden, L. (2014) Querying Dynamic Communities in Online Social Networks. Journal of Zhejiang University-SCIENCE C (Computers \& Electronics), 15, 81-90. http://dx.doi.org/10.1631/jzus.C1300281

[12] Wu, Y. (2015) Introduction of Timeline of Bibliometrics by Rousseau. Chinese SCIENCENET Blog. http://blog.sciencenet.cn/blog-1557-874940.html

[13] Zhou, T. (2015) Introduction of the Advance in the Research on Routing Strategy of Networks. Chinese SCIENCENET Blog. http://blog.sciencenet.cn/blog-3075-876277.html

[14] Cheng, C., Guo, Q. and Liu, J. (2015) Advance in the Research on Routing Strategy of Networks. Journal of University of Electronic Science and Technology of China, 44, 2-11.

[15] Weigang, L. (2015) Introduction of Rousseau and His Team's Study on Gefura Measures, Chinese SCIENCENET Blog. http://blog.sciencenet.cn/blog-652078-879702.html 